\title{
Online Auxiliaries for Instructors and Students
}

TEACHING GUIDES: UC Press website (www.ucpress.edu)

Studio Art - Level 1 - ECO ART GENRES

Art projects introduce bio art, digital art, installation, public art, social practice. Sample syllabus.

\section{Studio Art - Level 2 - ART STRATEGIES}

Art projects explore eco art visualization, intervention, activation, satirization. Sample syllabus.

\section{Contemporary Art History}

Assignments contextualize contemporary eco art within the history of art. Sample syllabus.

\section{Environmental Studies - Level 1 - ECO ART ISSUES}

Projects address energy, waste, climate change, habitat, sustainability. Sample syllabus.

\section{Environmental Studies - Level 2 - ECO APPROACHES}

Projects explore conservation, preservation, urban ecology, restoration ecology, deep ecology. Sample syllabus.

STUDENT RESOURCES: Author's website (www.lindaweintraub.com)
a. Color images of works discussed in the book.
b. Links to videos and artist interviews
c. Blog updating artists' careers and the themes they address. 
